\title{
Site-Directed Mutagenesis as a Tool for Unveiling Mechanisms of Bacterial Tellurite Resistance
}

\author{
José Manuel Pérez-Donoso ${ }^{1}$ and Claudio C. Vásquez² \\ 1 Universidad de Chile \\ ${ }^{2}$ Universidad de Santiago de Chile \\ Chile
}

\section{Introduction}

Tellurium (Te) is a scarce element in the earth's crust and is not essential for living organisms. It is rarely found in the non-toxic, elemental state $\left(\mathrm{Te}^{\mathrm{o}}\right)$; and the soluble oxyanions, tellurite $\left(\mathrm{TeO}_{3}{ }^{2-}\right)$ and tellurate $\left(\mathrm{TeO}_{4}{ }^{2-}\right)$, are toxic for most forms of life. Tellurite toxicity has been extensively exploited as a selective agent in diverse microbiological culture media.

A few bacterial tellurite resistance mechanisms have been proposed; but the genetic, biochemical and/or physiological bases underlying $\mathrm{TeO}_{3}{ }^{2-}$ resistance are still poorly understood.

One of our strategies to study bacterial resistance to $\mathrm{TeO}_{3}{ }^{2-}$ has been the cloning and characterization of genes from tellurite-resistant bacteria using Escherichia coli as a sensitive host. Using this experimental approach, we have previously shown that the genes $c y s K$, iscS and cobA [encoding cysteine synthase, cysteine desulfurase and S-Adenosyl-Lmethionine:uroporphirin-III C-methyltransferase (SUMT), respectively] from the thermophilic rod Geobacillus stearothermophilus V mediate tellurite resistance when expressed in E. coli. All of these genes were subjected to site-directed mutagenesis to demonstrate their participation in tellurite resistance in this mesophilic host (Vásquez et al., 2001; Tantaleán et al., 2003; Araya et al., 2009). More recently we conducted similar mutagenesis experiments with the Aeromonas caviae ST lpdA gene, encoding dihydrolipoil dehydrogenase, and found that two amino acid residues are involved in the tellurite reductase branch-activity of this enzyme (unpublished data).

Site-directed mutagenesis, also referred to as site-specific or oligonucleotide-directed mutagenesis, is a technique in molecular biology that allows the creation of mutations at a defined DNA sequence. In general, a synthetic primer containing the desired base change is hybridized to a single-stranded DNA containing the gene of interest; the rest of the gene is then copied using a DNA polymerase. The double-stranded DNA molecule thus obtained is ligated to an appropriate vector and introduced into a host cell for mutant selection.

This chapter does not intend to be an extensive review of tellurite resistance. Instead it was written as an example to make young scientists see how simple observations can help to 
state the basis of much more complex networks underlying a particular, defined phenomenon.

\section{The enigma of tellurite toxicity}

The ability of bacteria to counteract the effect of heavy metals has interested microbiologists for many years. Toxic heavy metals are often encountered in nature in many different forms. In air, they exist as metal or oxide dust. In surface and ground water, they are found attached to humic substances; and they also bind to soil and sediments.

Tellurium has applications in the semiconductor industry and electronics (in the production of thermoelements, photoelements and other devices in automation equipment). The increasing demand for new and different semiconductors necessitates research work on the application of various tellurium compounds as semiconductor components.

As a group, microorganisms display resistance to nearly all metal and non-metal ions that are considered toxic to the environment, including $\mathrm{Ag}^{+}, \mathrm{As}^{3+}, \mathrm{Cd}^{2+}, \mathrm{Cr}^{3+}, \mathrm{Hg}^{2+}, \mathrm{Sb}^{3+}, \mathrm{Te}^{4+}$, $\mathrm{Te}^{6+}$ and $\mathrm{Zn}^{2+}$, among others (Silver, 2006). Although the literature on the subject is vast and continuously updated (Silver, 2011), in most cases, however, the knowledge of the biochemical and/or genetic mechanisms underlying the metal resistance phenomena is still very limited. This is particularly true for bacterial tellurite resistance, in which much effort has been expended to understand how bacteria counteract the toxic effects of the tellurium salt.

Tellurium (Te) was considered almost an exotic element and was treated with certain indifference by most serious chemists. However, the impressive number of publications on Te compounds during the last few years shows that Te is now widely used in applied chemical reactions.

The natural Te cycle has not been investigated in depth, and the role of microbes - if any - in this process has not yet been elucidated. Nevertheless, tellurite-resistant bacteria do exist in nature; and they often reduce tellurite to its elemental, less toxic, form $\left(\mathrm{Te}^{\mathrm{o}}\right)$, which accumulates as black deposits inside the cell (Taylor, 1999; Chasteen et al., 2009).

As a result of the accumulated knowledge, several tellurite-resistance determinants $\left(\mathrm{Te}^{\mathrm{R}}\right)$ have been localized on plasmids and on the chromosome. Structure and organization vary greatly among bacterial species (Taylor, 1999). It has been argued that tellurite toxicity results from the ability of tellurite to act as a strong oxidizing agent that damages a number of cell components (Taylor, 1999; Pérez et al., 2007). In the last years, however, available evidence shows that tellurite toxicity results from the generation of reactive oxygen species (ROS) (Borsetti et al., 2005; Calderón et al., 2006; Tremaroli et al., 2007; Pérez et al., 2007). ROS, such as hydrogen peroxide $\left(\mathrm{H}_{2} \mathrm{O}_{2}\right)$, superoxide anion $\left(\mathrm{O}_{2}{ }^{-}\right)$and hydroxyl radical $\left(\mathrm{OH}^{*}\right)$, are typical byproducts of aerobic metabolism. However, they can also be produced upon exposure of the cell to free radical-generating compounds, like metals and metalloids.

Our group has been interested in studying tellurite resistance $\left(\mathrm{Te}^{\mathrm{R}}\right) /$ toxicity for many years. First we focused on thermophilic, Gram-negative, rods of the genus Thermus and later on G. stearothermophilus V, a thermotolerant, spore-forming, Gram-positive bacterium that was isolated in our laboratory from a soil sample. In both cases we demonstrated the existence of 
cellular reductases that convert tellurite into elemental tellurium at the expense of $\mathrm{NAD}(\mathrm{P}) \mathrm{H}$ oxidation in vitro (Chiong et al., 1988; Moscoso et al., 1998).

In an attempt to identify genetic determinants for $\mathrm{Te}^{\mathrm{R}}$ in these bacteria, we constructed gene libraries that were used to transform sensitive E. coli hosts to tellurite resistance. While the cloning of resistance determinants from Thermus has been unsuccesful so far, we did clone tellurite resistance determinants from $G$. stearothermophilus $\mathrm{V}$ into E. coli. These genes were subjected to site-directed mutagenesis in order to unveil their participation in the resistance phenomenon (Vásquez et al., 2001; Tantaleán et al., 2003; Araya et al., 2009). More recently, we have shown that overproduction of the Aeromonas caviae ST dihydrolipoil dehydrogenase results in enhanced tellurite resistance in E. coli. This enzyme exhibits NADH-dependent tellurite reductase (TR) activity (Castro et al., 2008, 2009). The change of two defined amino acid residues at the enzyme active site decreased TR activity (unpublished data). What follows is a chronological description of the above-mentioned results.

\subsection{CysK}

Cysteine synthases (CysKs) are enzymes that catalyze the last step in cysteine biosynthesis. They have been related to tellurite resistance in different microorganisms (Moore \& Kaplan, 1992; O'Gara et al., 1997; Alonso et al., 2000; Vásquez et al., 2001; Lithgow et al., 2004). All cysteine synthases described to date require the cofactor pyridoxal 5'-phosphate (PLP) for activity. PLP-dependent enzymes catalyze a broad spectrum of aminoacid transformations involved in the development of an organism, such as transaminations, $\beta$-eliminations, $\beta-\gamma$ replacements and racemizations.

Searching for tellurite-resistance determinants, we identified and characterized a new thermophilic CysK in G. stearothermophilus V (Saavedra et al., 2004). E. coli ovexpressing this cysK gene shows a tellurite resistance that is over 10-fold more than that observed for the wild-type controls. Despite the fact that it is known that the ping-pong catalytic mechanism of this enzyme is similar to that of other CysKs, the G. stearothermophilus V enzyme has not been fully characterized. The catalytic amino acid residues are also not known. In addition, the importance of cysteine synthase in bacterial tellurite resistance has not been totally documented; and our group has proposed that the resistance increase is paralleled by increased levels of reduced thiols, such as glutathione.

G. stearothermophilus V CysK is a homodimer (32 kDa/monomer) that requires one PLP molecule per subunit (Saavedra et al., 2004). It belongs to the $\beta$ family of PLP-dependent enzymes and shares some similarities with other enzymes involved in deamination reactions, as do tryptophan synthase, threonine deaminase and O-acetyl-serine sulphydrilase (Alexander et al., 1994).

A general mechanism for the CysK-catalyzed reaction has been proposed (Cook and Wedding, 1977; Tai et al., 1998). The enzyme binds PLP by a lysine group forming a Schiff base, known as internal aldimine. This intermediate absorbs in the $400-430 \mathrm{~nm}$ region and exhibits two resonant forms. Addition of the O-acetyl-L-serine (OAS) substrate allows the formation of the geminaldiamine intermediate, which produces the external aldimine. Then the quinonoid intermediate is formed; and when the substituent in the $\beta$ position is released (acetate), the $\alpha$-aminoacrylate is finally formed. 
To study the residues and motifs that define the catalytic properties of this enzyme, we used site-directed mutagenesis to assess the importance of the C-terminus and of some putative catalytic residues for CysK activity and CysK-mediated bacterial tellurite resistance (unpublished data).

As a first approach, a set of CysK C-terminal deletions of 10 (CysK $\Delta$ Tyr298), 20 (CysK $\Delta$ Leu288), 30 (CysK $\Delta$ Ala278), 40 (CysK $\Delta$ Gly268) and 60 (CysK $\Delta$ Ala248) amino acids were constructed, overexpressed, purified and characterized. Binding of the PLP cofactor was evaluated through the absorption spectrum of the purified proteins. An absorbance peak at $412 \mathrm{~nm}$ is characteristic of the $\alpha$-aminoacrylate intermediate.

All the CysK deletion mutants larger than $\triangle$ Tyr298 were inactive, unable to bind PLP and did not confer tellurite resistance. This result indicated a direct relationship between enzymatic activity and tellurite tolerance. It is also in agreement with a role for thiols, such as cysteine, in tellurite tolerance. In this context, increased levels of intracellular reduced thiols, particularly glutathione, were observed in cells overproducing CysK. This observation suggested that increased concentrations of cell antioxidants could be responsible for protecting cysK-overexpressing cells from tellurite-mediated oxidation. Interestingly, the mutant having Tyr298 (Tyrosine as residue 298, Y298) (see Table 1 in the chapter by Figurski et al. for the amino acid codes) as the C-terminal residue [CysK $\Delta$ (Tyr298)] was inactive, despite retaining the ability to bind PLP and to form the $\alpha$ aminoacrylate intermediate. As expected, overproduction of this mutant enzyme did not enhance tellurite resistance in $E$. coli.

As shown in Fig. 1, CysK displays the conserved amino acid sequence motif SVKDRIA near the amino terminus, which is required for PLP binding. Most of the C-terminal truncated mutants were unable to bind PLP, despite the presence of this motif and the finding that the proteins were correctly translated and folded in the cytoplasm. This observation suggests that other residues are involved in stabilizing PLP binding. The residues are probably located near the CysK C-terminus.

As can be deduced from these results, protein deletions allow a global view about the importance of some protein motifs and not a detailed interpretation on the participation of defined amino acids in the enzyme's functioning. In this context, site-directed mutagenesis offers a more versatile alternative to study the role of a defined motif or amino acid residue. These two experimental approaches can complement each other in order to obtain a detailed analysis for understanding the enzymatic mechanism.

To choose the appropriate residues to be subjected to site-directed mutagenesis, the first approach involved sequence conservation studies using BLASTP and ClustalW software and other programs. However, a better idea about which domains and/or residues could be interacting with defined molecules or atoms in a reaction can be obtained by constructing $3 \mathrm{D}$ models of the protein. In those cases where there is no crystallographic information regarding the protein, bioinformatic tools can offer a useful alternative in order to predict a model based on sequence homology.

Sequence analysis allowed identification of the conserved ${ }^{3}$ SVKDRIA49 domain (NCBI accession number AAG28533.1, see Fig. 1) in the G. stearothermophilus V CysK. In this conserved sequence, K45 is required to form the protonated Schiff base with PLP. K45 also 


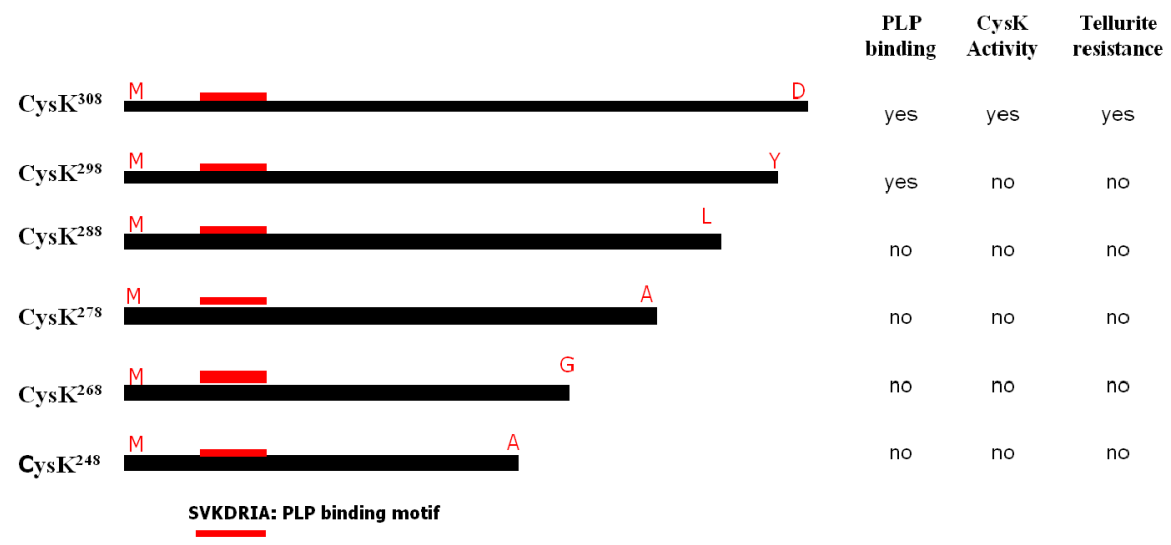

Fig. 1. Schematic model representing the different truncated CysKs and their properties (see the text for details).

participates directly in binding the cofactor and $\mathrm{NH}_{3}$ transamination. A replacement of $\mathrm{K} 45$ by alanine (K45A) was made in CysK to evaluate the importance of the positive charge required for PLP interaction. The mutated gene was overexpressed in E. coli, and the enzyme was purified to homogeneity. No enzymatic activity or cofactor binding was observed by the purified enzyme, confirming that this residue is required for PLP attachment, as predicted.

No structural data are available for G. stearothermophilus V CysK, so a 3D model based on the available crystallographic structure $\left(2 \mathrm{~A}^{\circ}\right.$ resolution) for the Salmonella enterica serovar Typhimurium CysK was constructed. With this model, we expected to make predictions about residues located at the enzyme's C-terminus that could be interacting with PLP, stabilizing it and allowing the transamination reaction.

As mentioned before, the deletion mutant CysK $\Delta$ (Tyr298) was inactive, but still able to bind PLP. Based on this observation, a homology model of this deletion mutant was constructed to define the position of specific residues that can participate in PLP stabilization both in wild-type CysK and in the CysK $\Delta$ (Tyr298) mutant.

The model suggested that the CysK $\Delta$ (Tyr298) deletion mutant exhibits amino acids in positions that can interact with PLP so as to orientate the cofactor for the reaction to proceed (Fig. 2). Tyrosine 298 is most probably interacting directly with PLP, given that it is located at a distance (less than $4 \mathrm{~A}^{\circ}$ ) that allows the formation of a hydrogen bond with the cofactor. Experiments with this deletion mutant confirmed that the enzyme was able to bind PLP and was folded correctly. However, studies performed to assess PLP- mediated fluorescence indicated that the cofactor displayed a different orientation than that in wild-type enzyme. Altogether, these results suggested that the Tyr298 residue could be important for CysK activity. Most probably Tyr298 forms a hydrogen bond through the hydroxyl group of the tyrosine, thus stabilizing and favoring a correct orientation of PLP. To test this possibility, homology analyses and new models of Y298A and Y298P CysK mutants were constructed to 
predict the role of the Tyr298 residue. The results suggested that the -OH group of Tyr298 is required for PLP binding. It is thought to affect catalysis because of an interaction with the $\alpha$-aminoacrylate intermediate through a water molecule.

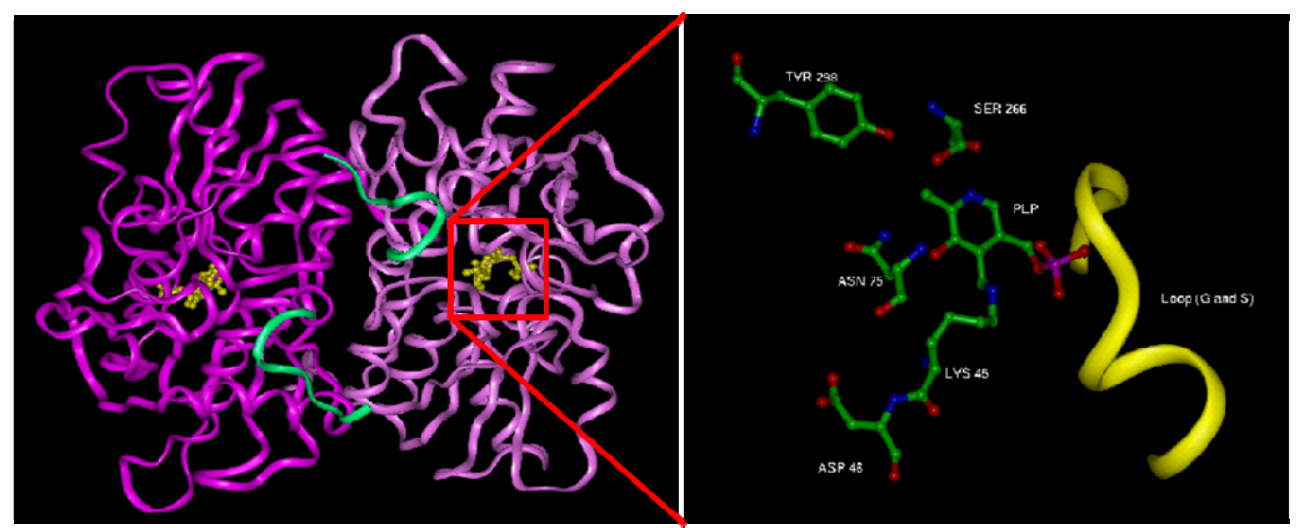

Fig. 2. Model (homology) of the G. stearothermophilus V CysK. Left, CysK homodimer showing one PLP molecule (yellow) bound per subunit and the C-terminal 10 amino acids (green). Right, schematic view of those amino acid residues involved in PLP binding (LYS45) and in orienting and/or stabilizing the cofactor (SER266, TYR298, and ASN75).

Two additional Tyr298 site-directed variants were constructed and characterized (Y298A and Y298F). Mutant proteins were purified and assayed for PLP binding, $\alpha$-aminoacrylate intermediate formation, and enzymatic activity (lyase and cysteine synthase). Both mutant enzymes were inactive and did not bind PLP nor formed the $\alpha$-aminoacrylate intermediate, suggesting that the -OH group of Tyr298 is required for CysK activity.

Based on these observations, we propose that the Tyr298 residue of G. stearothermophilus V CysK is involved in stabilization and proper orientation of PLP by interacting with the phosphate group of the cofactor through hydrogen bonding. The expression of the $G$. stearothermophilus $\mathrm{V}$ cysK gene was later shown to mediate low-level tellurite resistance in $E$. coli (Vásquez et al., 2001).

\subsection{IscS}

One of the superoxide anion targets is a family of dehydratases that use exposed [4Fe-4S] clusters to bind and dehydrate their substrates during the biosynthesis of branched-chain amino acids. Oxidation of these proteins results in the dismantling of these [Fe-S] centers with the concomitant loss of enzyme activity. Crude $E$. coli extracts catalyzing the formation of these centers in vitro contain at least four enzymatic activities that provide the required sulfur atoms: O-acetyl-serine sulfhydrilase A (CysK), O-acetyl-serine sulfhydrilase B (CysM) and $\beta$-cistationase; the fourth protein displayed cysteine desulfurase activity. Cysteine desulfurases remove the sulfur atom from cysteine to construct and repair [Fe-S] clusters in protein substrates that, in turn, catalyze essential redox reactions in critical metabolic pathways. Very soon it became clear that the IscS cysteine desulfurase played an important role in transferring the sulfur from cysteine for [Fe-S] center synthesis in vivo. 
Various tellurite-resistant $\left(\mathrm{Te}^{\mathrm{R}}\right)$ E. coli clones were isolated upon transformation with a G. stearothermophilus V HindIII library. In particular, one contained a 3.5 kilobase $(\mathrm{kb})$ DNA insert that specified three open reading frames (ORFs). By comparison with sequences deposited in protein data banks, it was found that ORF2 [1200 base pairs (bp)] encoded a $\sim 45 \mathrm{kDa}$ cysteine desulfurase (IscS). The expression of the G. stearothermophilus V IscS cysteine desulfurase conferred tellurite resistance in E. coli. The enzyme was induced and purified to homogeneity; the purified enzyme displayed cysteine desulfurase activity. We showed that tellurite resistance depends in part on the activity of the IscS enzyme, supporting the hypothesis that essential proteins with iron-sulfur [Fe-S] clusters are among the main targets of the oxidative damage caused by tellurite in E. coli. Unlike the case for other microbes, the G. stearothermophilus $\mathrm{V}$ iscS gene does not appear to be within an operon containing other genes involved in de novo [Fe-S] cluster formation.

Because G. stearothermophilus $\mathrm{V}$ is a thermophile, IscS purification included an incubation of soluble cell extracts at $70{ }^{\circ} \mathrm{C}$ for $20 \mathrm{~min}$. This step eliminated almost $75 \%$ of the total starting protein, without an appreciable loss of IscS protein or cysteine desulfurase activity (Fig. 3, lane 1). Subsequent column chromatography steps resulted in enzyme preparations that were $>98 \%$ pure. The amino-terminus of the purified IscS (MNLEQIRKDTPLHKKYSYIN), determined by Edman degradation, matched precisely the predicted primary sequence of the product of the iscS gene. The native form of the enzyme is a homodimer with an apparent molecular mass of $93-97 \mathrm{kDa}$, as determined by size-exclusion chromatography. This cysteine desulfurase belongs to the $\alpha$-family of PLP-dependent enzymes and exhibits an absorbance maximum for PLP centered at about $420 \mathrm{~nm}$, the characteristic UV-visible spectrum of other cysteine desulfurases.

To further confirm that IscS activity was responsible for tellurite resistance in E. coli, a series of mutant derivatives was constructed. Plasmids containing truncated versions of the iscS gene (90, 150 or 210 bp deletions of the $3^{\prime}$ end) did not confer resistance to tellurite in E. coli; nor did they exhibit desulfurase activity in crude extracts. The induced mutant proteins formed inclusion bodies, suggesting that the carboxyl terminus of IscS is essential for proper folding, dimerization and/or function.

We decided to make a directed change of Lys213, a residue that likely binds the PLP cofactor. This lysine is conserved in the sequence of cysteine desulfurases from both Grampositive and Gram-negative bacteria, as well as in the yeast Saccharomyces cerevisiae. Lys213 in IscS was replaced by alanine to yield the iscS_K213A mutant gene, which was cloned into the expression plasmid pET21b and introduced into E. coli JM109(DE3) to induce high-level transcription from the promoter. Cells expressing iscS K213A did not exhibit tellurite resistance.

The K213A enzyme was purified to homogeneity, using the same procedure as for the native enzyme, but omitting the initial heat-treatment because the mutant protein did not show the thermostability of the wild-type IscS (Fig. 3). Unlike extracts containing IscS, those of the IscS K213A mutant protein did not exhibit the typical intense yellow colour, consistent with the idea that Lys213 is critical for PLP binding. In fact, the absorbance peak characteristic of PLP-containing enzymes was missing in the UV-visible spectrum of the purified mutant protein. The mutant IscS enzyme showed less than $10 \%$ of the specific activity exhibited by the wild-type IscS. 


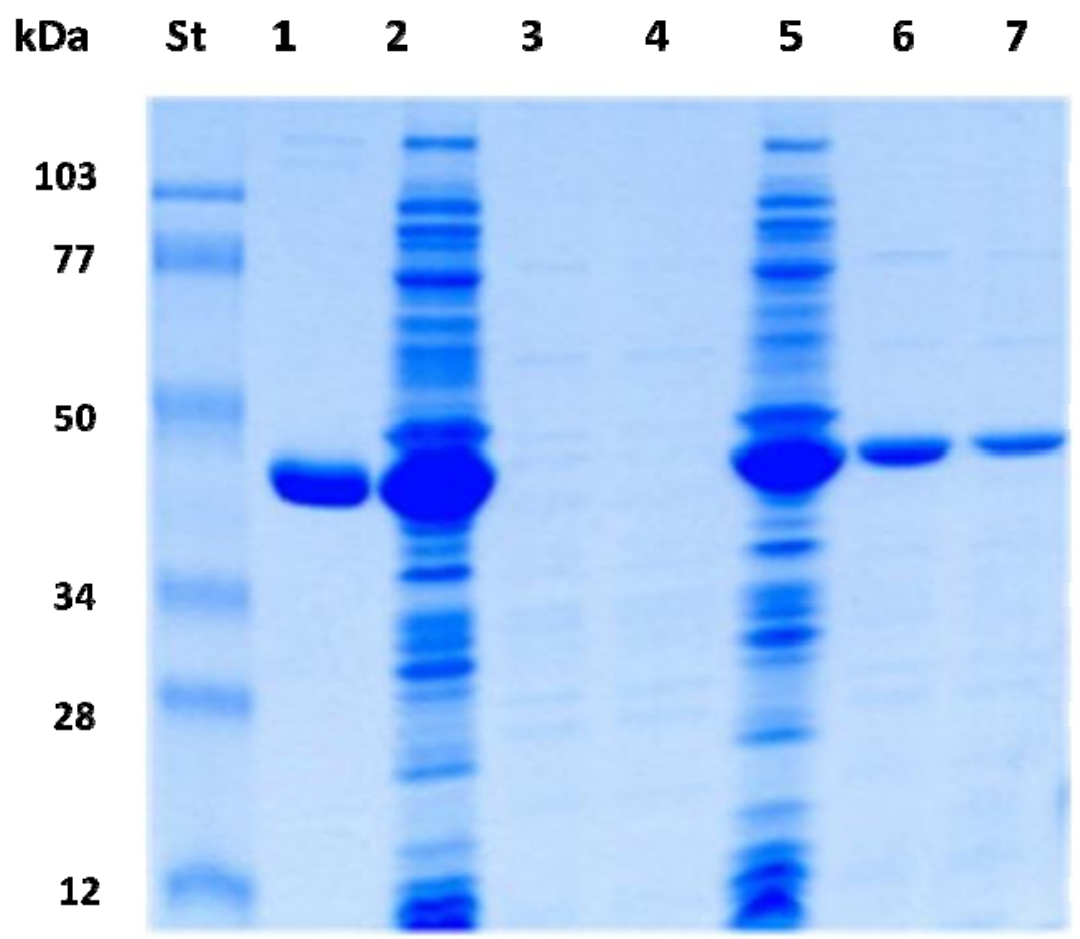

Fig. 3. Effect of temperature on the IscS K213A and K213R mutants. Lane 1, wild-type IscS. Lanes 2, 3 and 4, crude extracts of $E$. coli overproducing the K213A mutant cysteine desulfurase incubated for $10 \mathrm{~min}$ at $37^{\circ} \mathrm{C}, 10 \mathrm{~min}$ at $70^{\circ} \mathrm{C}$ and $20 \mathrm{~min}$ at $70{ }^{\circ} \mathrm{C}$, respectively. Lanes 5, 6, and 7, as in lines 2-4, but using E. coli extracts overproducing the K213R mutant cysteine desulfurase.

On the other hand, an IscS K223R mutant was also constructed to confirm the importance of the positive charge in protein stabilization, PLP binding and desulfurase activity. As shown in Figs. 3 and 4, the K213R IscS displayed the same thermostability behavior of the wildtype desulfurase; and the enzyme was also easily purified by heating. In addition, the purified K213R mutant displayed the characteristic intense yellow color of PLP enzymes, an observation that was further confirmed by the presence of the $412 \mathrm{~nm}$ absorbance peak in the UV-visible spectra. As expected, this mutant displayed desulfurase activity levels identical to those of the wild-type desulfurase (Fig. 4). These results confirmed that the arginine residue was able to maintain IscS function, indicating that the positive charge at this position is required for proper PLP binding (Fig. 5).

The G. stearothermophilus $\mathrm{V}$ iscS gene not only complemented successfully an E. coli iscS mutation, but also conferred tellurite resistance to an E. coli $\operatorname{sodAsodB}$ double mutant, arguing that superoxide causes specific damage to one or more critical [Fe-S] clustercontaining proteins. 


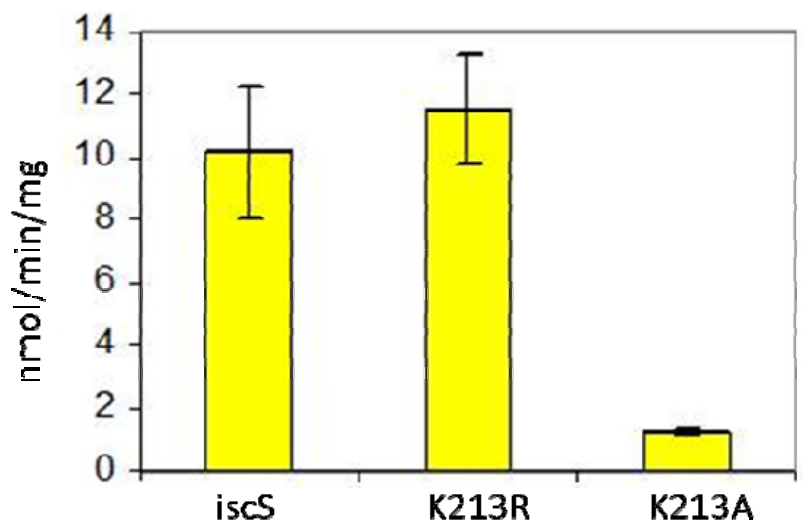

Fig. 4. Desulfurase activity of wild-type IscS and the indicated mutant IscSs.

\subsection{CobA}

A third gene mediating tellurite resistance in E. coli was identified from a G. stearothermophilus V HindIII library. The cobA gene was initially identified as one of three main ORFs present in a $3.8 \mathrm{~kb}$ G. stearothermophilus V DNA insert in the recombinant plasmid p1VH. E. coli carrying p1VH exhibited over 10 fold the resistance to potassium tellurite observed in the same strain harboring the pSP72 cloning vector alone (Araya et al., 2004).
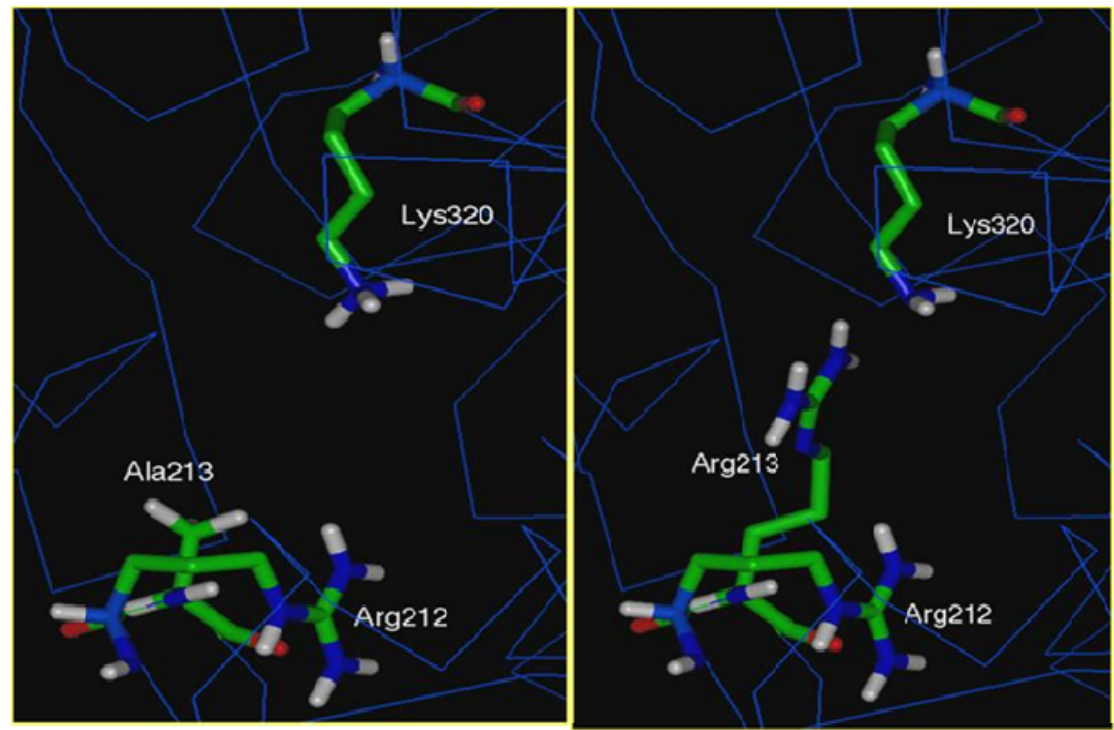

Fig. 5. Molecular models of the active site of the K213A and K213R mutants of the IscS enzyme from G. stearothermophilus V. 
The cobA gene of G. stearothermophilus $\mathrm{V}$ encodes a $28 \mathrm{kDa}$ protein exhibiting $71 \%$ identity with the Bacillus megaterium cobA gene, which encodes the enzyme S-adenosyl-Lmethionine:uroporphirin-III C-methyltransferase, also referred to as SUMT.

The cobA gene was amplified by PCR using appropriate primers, inserted into the pET21b expression vector and introduced into E. coli JM109(DE3). Transformants exhibited higher tellurite resistance than that observed for cells carrying the cloning vector alone. Minimal inhibitory concentration (MIC) determinations were carried out in the absence of the inducer (IPTG), since the protein seemed to be toxic to the cell when overexpressed.

G. stearothermophilus V SUMT was induced with IPTG and judged $>95 \%$ pure after being purified in two chromatographic steps (Cibacron blue and Sephadex column chromatography). After being fractionated by polyacrylamide gel electrophoresis in the presence of sodium dodecyl sulphate (PAGE-SDS) and transferred to a polyvinylidene fluoride (PVDF) membrane, the purified SUMT was sent for EDMAN microsequencing analysis. The first 10 amino acids (MTNGKVYIVG) matched $100 \%$ of those predicted by the nucleotide sequence of the $\operatorname{cobA}$ gene. A $M r$ of $60 \mathrm{kDa}$, compatible with a homodimeric quaternary structure, was deduced for the SUMT enzyme by electrophoresis under nondenaturing conditions and by gel chromatography.

The cobA-encoded amino acid sequence was compared with that of other methylases involved in corrinoid biosynthesis. Highly conserved regions were present in all analyzed sequences. One of them, whose consensus is GXGXGD, has been described as a SAMbinding motif (Fig. 6), suggesting that the G. stearothermophilus $V \operatorname{cobA}$ gene product is actually an uroporphirinogen III-like C-methyltransferase. Appropriate primers were designed to introduce a change of $A$ to $G$ at position 12 of the SUMT enzyme by recombinant PCR. The product was cloned into the pET21b(+) expression vector and the A12G mutant protein was purified as above. As the wild-type counterpart, the SUMT A12G protein exhibited a homodimeric structure, as determined by non-denaturing polyacrylamide gel electrophoresis and size exclusion chromatography. Cells expressing the mutant SUMT showed low $\mathrm{K}_{2} \mathrm{TeO}_{3}$ resistance (MIC $2.5 \mu \mathrm{g} / \mathrm{ml}$ ) as compared to the wild-type clone (MIC $18 \mu \mathrm{g} / \mathrm{ml}$ ).

Various deletion mutants of the cobA gene were constructed by PCR that included 60-, 120and 180-bp deletions from its $3^{\prime}$ end. The truncated DNA fragments were amplified and cloned into the pET21b(+) expression vector. Plasmids carrying cobA $\Delta 60, \operatorname{cob} A \Delta 120$ and cobA $\Delta 180$ were introduced into E. coli JM109(DE3) by transformation. Although expressed in high amounts, purification of the truncated proteins failed, as they formed inclusion bodies. Several attempts to solubilize them were carried out also without results. All clones expressing truncated genes were sensitive to tellurite (MIC $1.25 \mu \mathrm{g} / \mathrm{ml}$ ).

Addition of methyl- ${ }^{3} \mathrm{H}$ SAM to the purified SUMT enzyme followed by size exclusion chromatography revealed two radioactive peaks, corresponding to enzyme-bound and free SAM. When the wild-type enzyme was replaced by the SUMT A12G mutant in the SAM binding assay, only the free SAM peak was observed.

Another strategy of a cell to cope with the toxic effects of tellurite is to form volatile, less toxic, compounds with it. In this context, headspace analysis from cultures of different bacteria by gas chromatography- fluorine induced chemiluminescence detection (GC- 
F2ICD) has proven to be useful for detecting the evolution of sulfur compounds, such as methanethiol (MeSH), dimethyl sulfide (DMS), dimethyl disulfide (DMDS), dimethyl trisulfide (DMTS), and organotellurides, like dimethyl telluride (DMTe) (Chasteen and Bentley, 2003).

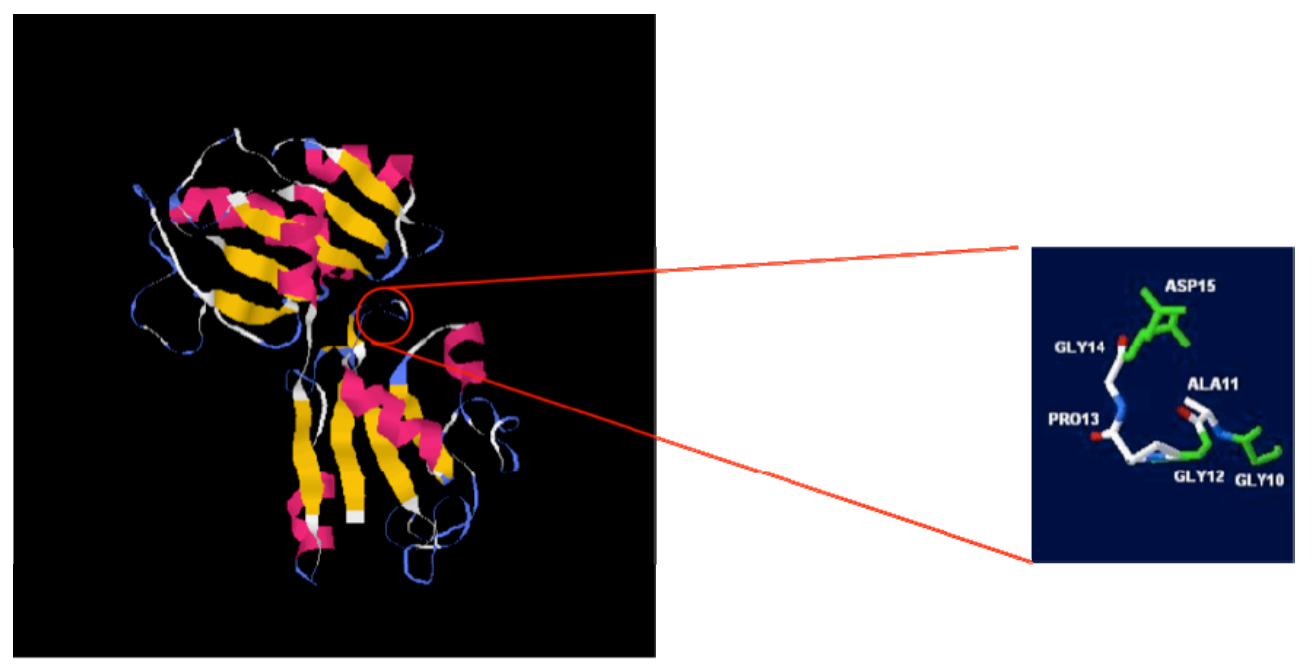

Fig. 6. Model (homology) of the SUMT methyl transferase. The inset shows the SAMbinding motif.

Given that SUMT is a methyltransferase, it was tempting to correlate it a priori with the resulting volatile Te derivatives. Since methylcobalamins (methyl-B12) have been involved in biomethylating a number of heavy metals and metalloids, one putative way by which SUMT could participate in tellurite resistance in E. coli could be precisely through this kind of methylation. However, two lines of evidence allow this assumption to be discarded. First, E. coli does not synthesize methyl-B12 de novo; and, second, amending the culture medium with $10 \mathrm{mM} \mathrm{CoCl}_{2}$ did not change the $\mathrm{K}_{2} \mathrm{TeO}_{3} \mathrm{MIC}$. It is well known that Co salts inhibit any methyl-B12-mediated methylation. Thus, a putative role of SUMT in $\mathrm{K}_{2} \mathrm{TeO}_{3}$ resistance would be the utilization of Te as a substrate and to catalyze the transfer of methyl groups from SAM to the metalloid. In this context, some work from other authors has indicated that the gene products of the tpm and tehB genes from Pseudomonas syringae and E. coli, respectively, are able to biomethylate tellurium (Cournoyer et al., 1998; Liu et al., 2000). Unfortunately, we were unable to detect the genesis of methylated tellurium derivatives in the headspace of cells cultured in the presence of tellurite or tellurate. To date there is no clear experimental evidence that sheds light on the enzymatic mechanism underlying tellurium biomethylation.

On the other hand, since sulfite reductase (reduces sulfite to sulfur) utilizes siroheme as a prosthetic group, SUMT could participate in tellurite tolerance by enhancing the biosynthesis of this cofactor and, hence, that of cysteine. In the same context, it was found that enzymes that reduce thiols (glutathione and thioredoxin reductases) and their metabolites (thioredoxins, glutaredoxins and glutathione) would be involved in tellurite 
resistance. Recent results from our laboratory indicate that when grown in the presence the toxicant, the total thiol content is higher in cells expressing the cobA gene than in cells carrying the vector alone. Our interpretation is that SUMT could participate in the generation of reducing power (cysteine, for example) that would be used to compensate (or to recover) GSH or another metabolically important thiol that could have been consumed during tellurite reduction.

\subsection{LpdA}

As mentioned before, one of the most relevant properties of potassium tellurite is its high toxicity for microorganisms. In this context, our approach to understand the basis of the toxic effects has been the search of resistance determinants in tellurite-resistant strains, such as G. staerothermpophilus V. Following the same idea, another highly $\mathrm{TeO}_{3}{ }^{2-}$-resistant bacterial strain was isolated from environmental water. This new strain exhibited a tellurite MIC close to $300 \mu \mathrm{g} / \mathrm{ml}$. It was identified as the Gram-negative Aeromonas caviae ST. In addition to its high resistance to tellurite, this strain exhibited high levels of tellurite reduction, as determined by the darkness of cells exposed to the toxic salt and by tellurite reductase (TR) enzymatic assays performed with cell-free extracts. Interestingly, most of this TR activity was dependent of $\mathrm{NADH}$ and tracked to the pyruvate dehydrogenase multienzymatic complex (PDH), specifically to the E3 component encoded by the $1 p d A$ gene (Castro et al., 2008, 2009).

The lpdA gene was cloned; and the recombinant plasmid was used as template to construct three different mutants by site-directed mutagenesis: C45A, H322Y and E354K (Fig. 7). These mutants were chosen based on previous work on the E. coli $\mathrm{E} 3$ component indicating that C45 is highly conserved and is involved in the formation of a disulfide bond with C50, required for appropriate protein conformation (Kim et al., 2008). H322 and E354 were changed to $\mathrm{Y}$ and $\mathrm{K}$, respectively, because it was previously shown that these mutations affect NADH binding, a substrate required for PDH as well as for TR activity (Castro et al., 2008, 2009).

In this case a different and easier approach to construct the mutants was carried out. Using a high-fidelity and highly processive DNA polymerase and two complementary primers, the plasmid was amplified by PCR and then the methylated template was digested with DpnI restriction endonuclease and used to transform E. coli.

As expected, changes of these amino acids resulted in negative effects on pyruvate dehydrogenase activity in cells overproducing these proteins as compared to controls. Decreased PDH activity was observed, particularly in the cases of the H322Y and E354K mutants. The effect was not so pronounced in mutants that do not affect NADH binding. Regarding tellurite reductase activity (TR), an important decrease $(\sim 70 \%)$ was observed in all three mutants, as determined with purified proteins or in crude extracts of cells overproducing the respective mutant (Fig. 8). These results confirm the importance of NADH for PDH and TR activities and also indicated that C45, while relevant for LpdAmediated tellurite reduction, is not absolutely required for $\mathrm{PDH}$ activity. This idea is in agreement with previous observations of our group and others regarding the importance of cysteine in tellurite resistance (Vásquez et al., 2001; Fuentes et al., 2007). 


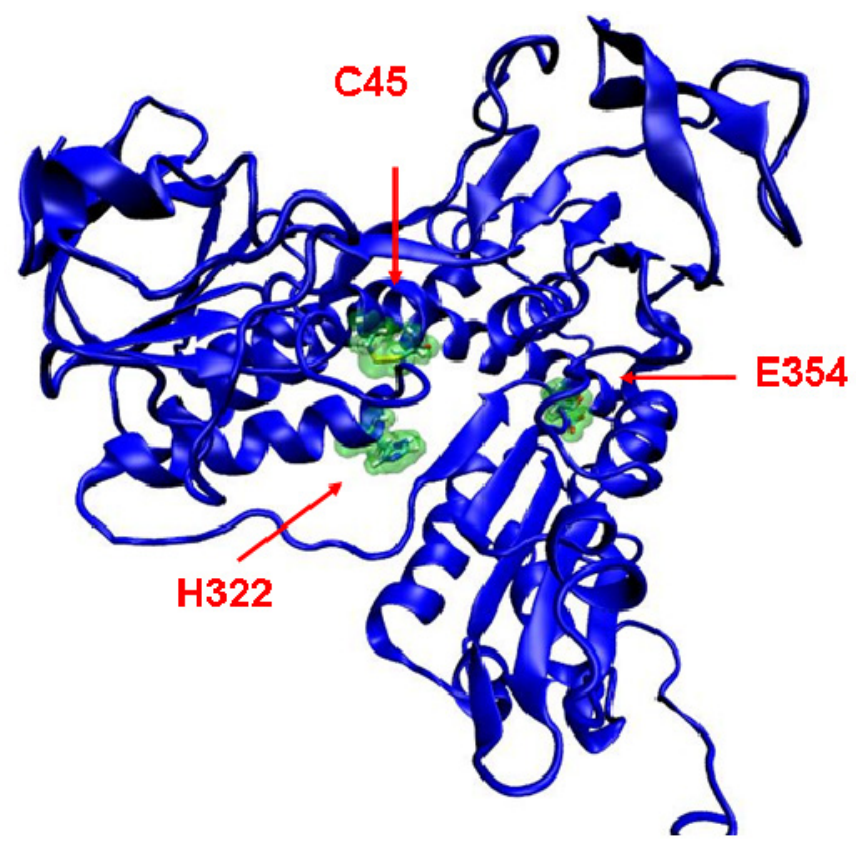

Fig. 7. LpdA model (homology) indicating the spatial position of the amino acids targeted for site-directed mutagenesis.

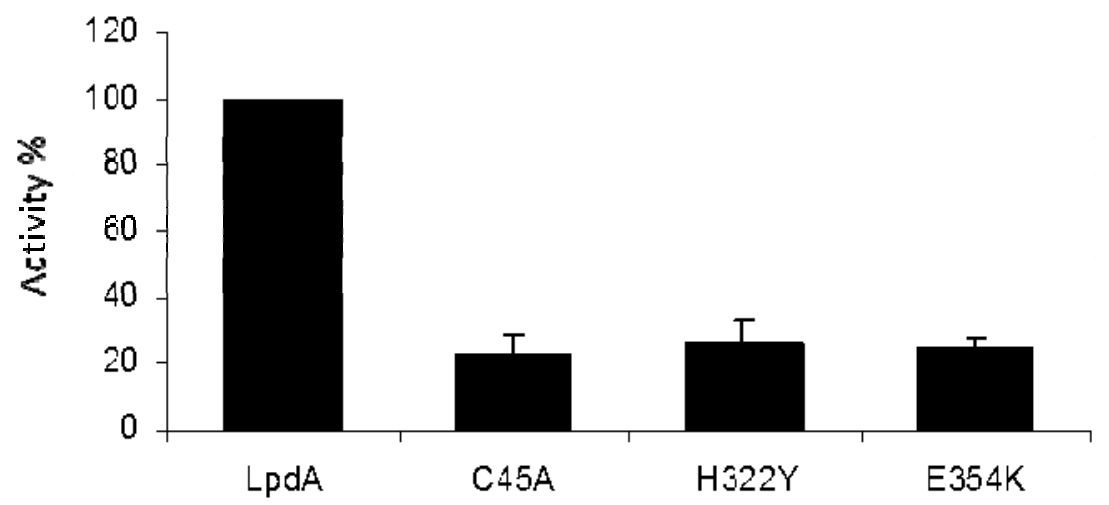

Fig. 8. Tellurite reductase activity of purified LpdA and the indicated LpdA mutants.

\section{Conclusion}

Several different mechanisms have been proposed to account for the toxicity of tellurite. Tellurium may replace sulfur and/or selenium in critical metabolites or enzymes and abate their essential functions. Alternatively, tellurite is a strong oxidizing agent that may cause general oxidative damage; or it may cause specific damage to critical thiol groups or [Fe-S] 
clusters present in essential enzymes. The results of this chapter point out different instances in which diverse metabolic pathways, their substrates or products play a still not welldefined role in bacterial tellurite resistance.

\section{Acknowledgments}

The authors thank Fondecyt grants \# 1090097 and 3100049 (Fondo de Desarrollo Científico y Tecnológico, Chile) and Dicyt-USACH (Dirección de Investigación en Ciencia y TecnologíaUniversidad de Santiago de Chile).

\section{References}

Alexander, F.W., Sandmeier, E., Mehta, P.K. \& Christen, P. (1994). Evolutionary relationships among pyridoxal-5'-phosphate-dependent enzymes. Regio-specific alpha, beta and gamma families. Eur. J. Biochem., Vol. 219, No. 3, (February 1994), pp. 953-960, ISSN 0014-2956

Alonso, G., Gomes, C., González, C. \& Rodríguez-Lemoine, V. (2000). On the mechanism of resistance to channel-forming colicins (PacB) and tellurite, encoded by plasmid Mip233 (IncHI3). FEMS Microbiol. Lett., Vol. 192, No. 2, (November 2000), pp. 257261, ISSN 0378-1097

Araya, M.A., Tantaleán, J.C., Fuentes, D.E., Pérez, J.M., Calderón, I.L., Saavedra, C.P., Chasteen, T.G. \& Vásquez, C.C. (2009). Cloning, purification and characterization of Geobacillus stearothermophilus V uroporphirinogen-III C-methyltransferase: evaluation of its role in resistance to potassium tellurite in Escherichia coli. Res. Microbiol., Vol. 160, No. 2, (March 2009), pp. 125-133, ISSN 0923-2508

Borsetti, F., Tremaroli,V., Michelacci, F., Borghese, R., Winterstein, C., Daldal, F. \&, Zannoni, D. (2005). Tellurite effects on Rhodobacter capsulatus cell viability and superoxide dismutase activity under oxidative stress conditions. Res Microbiol., Vol. 156, No. 7, (August 2005), pp. 807-813, ISSN 0923-2508

Calderón, I.L., Arenas, F.A., Pérez, J.M., Fuentes, D.E., Araya, M.A., Saavedra, C.P., Tantaleán, J.C., Pichuantes, S.E., Youderian, P.A. \& Vásquez, C.C. (2006). Catalases are $\mathrm{NAD}(\mathrm{P}) \mathrm{H}$-dependent tellurite reductases. PLoS ONE, Vol. 1, No. 1, (December 2006), pp. e70, ISSN 1932-6203

Castro, M.E., Molina, R., Díaz, W., Pichuantes, S.E. \& Vásquez, C.C. (2008). The dihydrolipoamide dehydrogenase of Aeromonas caviae ST exhibits NADH dependent tellurite reductase activity. Biochem. Biophys. Res. Commun., Vol. 375, No. 1, (October 2008), pp. 91-94, ISSN 0006-291X

Castro, M.E., Molina, R.C., Díaz, W.A., Pradenas, G.A. \& Vásquez, C.C. (2009). Expression of Aeromonas caviae ST pyruvate dehydrogenase complex components mediate tellurite resistance in Escherichia coli. Biochem. Biophys. Res. Commun., Vol. 380, No. 1, (February 2009), pp. 148-152, ISSN 0006-291X

Chasteen, T.G. \& Bentley, R. (2003). Biomethylation of selenium and tellurium: microorganisms and plants. Chem. Rev., Vol. 103, No. 1, (January 2003), pp. 1-25, ISSN 0009-2665

Chasteen, T.G., Fuentes, D.E., Tantaleán, J.C. \& Vásquez, C.C. (2009). Tellurite: history, oxidative stress and molecular mechanisms of resistance. FEMS Microbiol. Rev., Vol. 33, No. 4, (July 2009), pp. 820-832, ISSN 1574-6976 
Chiong, M., Barra, R., González, E. \& Vásquez C. (1988). Resistance of Thermus spp. to potassium tellurite. Appl. Environ. Microbiol., Vol. 54, No. 2, (February 1988), pp. 610-612, ISSN 0099-2240

Cook, P.F. \& Wedding R.T (1977). Overall mechanism and rate equation for O-acetylserine sulfhydrylase. J. Biol. Chem., Vol. 252, No. 10, (May 1977), pp. 3549-3553, ISSN 00219258

Cournoyer, B., Watanabe, S. \& Vivian, A. (1998). A tellurite-resistance genetic determinant from phytopathogenic pseudomonads encodes a thiopurine methyltransferase: evidence of a widely-conserved family of methyltransferases. Biochim Biophys Acta., Vol. 1397, No. 2, (April 1998), pp. 161-168, ISSN 0304-4165

Fuentes, D.E., Fuentes, E.L., Castro, M.E., Pérez, J.M., Araya, M.A., Chasteen, T.G., Pichuantes, S.E. \& Vásquez, C.C. (2007). Cysteine metabolism-related genes and bacterial resistance to potassium tellurite. J. Bacteriol., Vol. 189, No. 24, (October 2007), pp. 8953-8960, ISSN 0021-9193

Kim, Y., Ingram, L. \& Shanmugam, T. (2008). Dihydrolipoamide dehydrogenase mutation alters the NADH sensitivity of pyruvate dehydrogenase complex of Escherichia coli K-12. J. Bacteriol., Vol. 190, No. 11, (March 2008), pp. 3851-3858, ISSN 0021-9193

Lithgow, J.K., Hayhurst, E.J., Cohen, G., Aharonowitz, Y. \& Foster, S.J. (2004). Role of a cysteine synthase in Staphylococcus aureus. J. Bacteriol. Vol. 186, No. 6, (March 2004), pp. 1579-1590, ISSN 0021-9193

Liu, M., Turner, R.J., Winstone, T.L., Saetre, A., Dyllick-Brenzinger, M., Jickling, G., Tari, L.W., Weiner, J.H. \& Taylor, D.E. (2000). Escherichia coli TehB requires Sadenosylmethionine as a cofactor to mediate tellurite resistance. J. Bacteriol., Vol. 182, No. 22, (November 2000), pp. 6509-6513, ISSN 0021-9193

Moore, M. \& Kaplan, S. (1992). Identification of intrinsic high-level resistance to rare-earth oxides and oxyanions in members of the class Proteobacteria: characterization of tellurite, selenite, and rhodium sesquioxide reduction in Rhodobacter sphaeroides. J. Bacteriol., Vol. 174, No. 5, (March 1992), pp. 1505-1514, ISSN 0021-9193

Moscoso, H., Saavedra, C., Loyola, C., Pichuantes, S. \& Vásquez C. (1998). Biochemical characterization of tellurite-reducing activities of Bacillus stearothermophilus V. Res. Microbiol., Vol. 149, No. 6, (June 1998), pp. 389-397, ISSN 0923-2508

O'Gara, J., Gomelsky, M. \& Kaplan, S. (1997). Identification and molecular genetic analysis of multiple loci contributing to high-level tellurite resistance in Rhodobacter sphaeroides 2.4.1. Appl. Environ. Microbiol., Vol. 63, No. 12, (December 1997), pp. 4713-4720, ISSN ISSN 0099-2240

Pérez, J.M., Calderón, I.L., Arenas, F.A., Fuentes, D.E., Pradenas, G.A., Fuentes, E.L., Sandoval, J.M., Castro, M.E., Elías, A.O. \& Vásquez, C.C. (2007). Bacterial toxicity of potassium tellurite: unveiling an ancient enigma. PLOS ONE. Vol. 2, No. 2, (February 2007), pp. e211, ISSN 1932-6203

Saavedra, C.P., Encinas, M.V., Araya, M.A., Pérez, J.M., Tantaleán, J.C., Fuentes, D.E., Calderón, I.L., Pichuantes, S.E. \& Vásquez, C.C. (2004). Biochemical characterization of a thermostable cysteine synthase from Geobacillus stearothermophilus V. Biochimie, Vol. 86, No. 7, (July 2004), pp. 481-485, ISSN 0300-9084

Silver S. (1996). Bacterial resistances to toxic metal ions--a review. Gene, Vol. 179, No. 1, (November 1996), pp. 9-19, ISSN 0378-1119 
Silver, S. (2011). BioMetals: a historical and personal perspective. Biometals, Vol. 24, No. 3, (June 2011), pp. 379-390, OnlineISSN 1572-8773

Tai, C.H., Yoon, M.Y., Kim, S.K., Rege, V.D., Nalabolu, S.R., Kredich, N.M., Schnackerz, K.D. \& Cook, P.F. (1998). Cysteine 42 is important for maintaining an integral active site for O-acetylserine sulfhydrylase resulting in the stabilization of the alphaaminoacrylate intermediate. Biochemistry, Vol. 37, No. 30, (July 1998), pp. 1059710604, ISSN 0006-2960

Tantaleán, J.C., Araya, M.A., Saavedra, C.P., Fuentes, D.E., Pérez, J.M., Calderón, I.L., Youderian, P. \& Vásquez, C.C. (2003). The Geobacillus stearothermophilus V iscS gene, encoding cysteine desulfurase, confers resistance to potassium tellurite in Escherichia coli K-12. J. Bacteriol., Vol. 185, No. 19, (October 2003), pp. 5831-5837, ISSN 0021-9193

Taylor, D.E. (1999). Bacterial tellurite resistance. Trends Microbiol., Vol. 7, No. 3, (March 1999), pp. 111-115, ISSN 0966-842X

Tremaroli, V., Fedi, S. \& Zannoni, D. (2007). Evidence for a tellurite-dependent generation of reactive oxygen species and absence of a tellurite-mediated adaptive response to oxidative stress in cells of Pseudomonas pseudoalcaligenes KF707. Arch Microbiol., Vol. 187, No. 2, (February 2007), pp. 127-135, ISSN 1432-072X

Vásquez, C., Saavedra, C., Loyola, C., Araya, M. \& Pichuantes, S. (2001). The product of the cysK gene of Bacillus stearothermophilus $\mathrm{V}$ mediates potassium tellurite resistance in Escherichia coli. Curr. Microbiol., Vol. 43, No. 6, (December 2001), pp. 418-421, ISSN 1432-0991 